“ (C) 2019 IEEE. Personal use of this material is permitted. Permission from IEEE must be obtained for all other uses, in any current or future media, including

reprinting/republishing this material for advertising or promotional purposes, creating new collective works, for resale or redistribution to servers or lists, or reuse of any copyrighted component of this work in other works." 


\title{
Period adding bifurcations in dynamic pricing processes
}

\author{
Shuixiu Lu ${ }^{1,2}$, Sebastian Oberst ${ }^{1}$, Guoqiang Zhang ${ }^{1}$, Zongwei Luo ${ }^{2}$ \\ ${ }^{1}$ Centre for Audio, Acoustics and Vibration (CAAV), FEIT, University of Technology Sydney, NSW 2007, Australia \\ ${ }^{2}$ Department of Computer Science and Engineering, Southern University of Science and Technology, China
}

\begin{abstract}
Price information enables consumers to anticipate a price and to make purchasing decisions based on their price expectations, which are critical for agents with pricing decisions or price regulations. A company with pricing decisions can aim to optimise the short-term or the long-term revenue, each of which leads to different pricing strategies thereby different price expectations. The choices between the two optimisation objectives consider the maximal revenue and the robustness of a chosen pricing strategy against market volatility. However the robustness is rarely identified in a volatile market. Here, we investigate the robustness of optimal pricing strategies with the short-term or long-term optimisation objectives through the analysis of nonlinear dynamics of price expectations. Bifurcation diagrams and period diagrams are introduced to compare their change in dynamics. Our results highlight that period adding bifurcations occur during the dynamic pricing processes studied. These bifurcations would challenge the robustness of an optimal pricing strategy. The consideration of the long-term revenue allows a company to charge a higher price, which in turn increases the revenue. However, the consideration of the shortterm revenue can avoid period adding bifurcations, contributing to a robust pricing strategy. This allows a company to harvest a good revenue through a robust pricing strategy in a volatile market and to satisfy regulations of a control in price volatility.

Index Terms-Border collision bifurcations, optimal price choices, reference price
\end{abstract}

\section{INTRODUCTION}

Price transparency due to information technology, especially the internet, influences strongly on consumer purchasing decision [1]. Consumers tend to use online travel agents or meta-search engines to facilitate purchasing decisions of an airline ticket [2]. Price information, which can be conveniently accessed over the internet, allows consumers to compare the price from different channels and to anticipate a price as a reference point to support purchasing decisions [3].

When the anticipation of the price is based on historical prices, the price anticipation is called the internal reference price [4] or simply reference price in short.

The reference price acts as a benchmark for consumer purchasing decisions [3], [5]-[7]. While a sales price lower than a reference price is perceived by the customer as a gain, a sales price higher than the reference price is perceived as a loss. An experience of receiving a gain renders consumers to be more likely to make a purchasing decision. However, consumers who experience a loss tend to reject a purchase. The

Author emails: shuixiu.lu@student.uts.edu.au, sebastian.oberst@uts.edu.au guoqiang.zhang@uts.edu.au, luozw@sustc.edu.cn effect of the perceived gains/losses on the demand is called the reference effects [7].

Owing to reference effects, a decision maker with pricing decisions could set a lower price to attract a higher volume of consumers and to receive the increased short-term revenue. The decision maker, however, may suffer losses considering the long-term revenue: a low price decreases consumer reference price, which in turn makes them less likely to accept a higher price in the future [5].

Although the long-term revenue is critical, subjective reasons may influence the decision maker to emphasise the shortterm revenue such as the quarterly earning expectations or the subjective job security cf. [8].

Another reason to focus on the short-term revenue is the computational complexity found in the associated optimisation problem. An analytic solution is possible for the task optimising the short-term revenue [3], [9].However, when consumers behave asymmetrically towards gains and losses, a demand function becomes non-smooth, which challenges the optimisation of the long-term revenue. So far, no explicit analytic solution is yet available for this type of problem [3], [6], [9]. Only numerical approximations can be obtained [3], [9].

One natural question that arises from the analytic and computational advantages of the short-term revenue strategy is that:

Can a decision maker benefit from a short-term revenue strategy and simultaneously gain insights from its detailed analysis to guide the pricing decision process for the longterm revenue?

Answering the question includes two criteria to evaluate an optimal pricing strategy: the maximal revenue obtained from it and its robustness against the volatile market and optimsiation objectives. To date optimal pricing strategy between the goal of the short-term and the long-term revenue have already been compared regarding to the maximal revenue [5]. However, the comparison neglects the robustness of the optimal pricing strategy. Optimisation based on an altered environment, however, requires more than the revenue alone to be considered. Therefore, in practice, a decision maker needs the robustness to evaluate optimal pricing strategies except for the maximal revenue [10], [11].

Also, consumers asymmetrically respond to gains and losses [3], [5], [6]. This leads to two different tangent slopes at the point in the demand curve where the reference price equals the 
sales price in the demand function. As a result, the demand function is non-smooth.

Owing to the non-smoothness of the problem, border collision bifurcations have been reported in mathematical models underlying dynamic pricing processes [3]. Border collision bifurcations or grazing bifurcations are also widely observed in other systems such as impact or friction oscillators [12], [13], and financial market [14].

Period adding or period incrementing bifurcations are the cornerstones of border collision bifurcations [14]-[16]. For period incrementing bifurcations, the period either increases or decreases by a positive integer; for period adding bifurcations, the period follows the Farey summation rule with changing bifurcation parameter [14]-[16]. According to the Farey rule period- $m+n$ solution can been observed between the bifurcation parameters which generate period- $m$ and period- $n$ solution.

Although dynamic pricing models are generally nonlinear with a nonsmooth demand function, their bifurcation structures have not been studied to the best of the authors knowledge. Here we aim to uncover these structures for the short-term and long-term revenue and introduce the bifurcation and period diagrams to study the robustness of optimal pricing strategies and answer the abovementioned question.

\section{MODELS}

\section{A. Reference price}

Following a dynamic pricing process, the reference price evolves via

$$
r_{n+1}=\alpha r_{n}+(1-\alpha) p_{n}
$$

where $r_{n}$ is the reference price at period $n, p_{n}$ is the sales price at period $n$, and $\alpha \in[0,1]$ represents a memory factor.

For a given initial $r_{0}$, Eq. (1) can be transformed to

$$
r_{n+1}=\alpha^{n+1} r_{0}+(1-\alpha)\left(\alpha^{n} p_{0}+\alpha^{n-1} p_{1}+\cdots+\alpha^{0} p_{n}\right)
$$

In an extreme case when $\alpha=0$ in Eq. (2), consumers only remember the latest price; for $\alpha>0$ consumers start remembering past prices but slow down their adaptions to the past until for $\alpha=1$, consumers memorise only $r_{0}$. Here, being consistent with $\mathrm{Hu}$ et al. [3], we define $r_{0} \in[0, U]$ and $p_{n} \in[0, U]$ with $U$ being the maximal price that a decision maker is allowed to charge.

\section{B. Demand}

Following $\mathrm{Hu}$ et al. [3], the demand $d\left(p_{n}, r_{n}\right)$ is composed of a linear base demand and nonlinear reference effects via

$$
d\left(p_{n}, r_{n}\right)= \begin{cases}b-a p_{n}+\gamma^{g}\left(r_{n}-p_{n}\right), & \text { if } r_{n}>p_{n}, \\ b-a p_{n}, & \text { if } r_{n}=p_{n}, \\ b-a p_{n}+\gamma^{l}\left(r_{n}-p_{n}\right), & \text { if } r_{n}<p_{n} .\end{cases}
$$

Here, Eq. (3b) represents the base demand, $a$ and $b$ are parameters of the base demand, and $\gamma^{g}$ and $\gamma^{l}$ stand for the sensitivity of consumers to either a gain or a loss, respectively.

When $\gamma^{g} \neq \gamma^{l}$, the demand becomes non-smooth. Hu et al. [3] restricts their study to the special case of $\gamma^{l}=0$. Yet, this assumption can result in losses [3] and should therefore be avoided from a management point of view. A more general scenario would be required to better understand the dynamics and to avoid losses. We therefore define the new parameter $\beta$ to act as the relative sensitivity of consumers to losses and gains,

$$
\beta=\gamma^{l} / \gamma^{g}:
$$

- If $\beta=1$, then $\gamma^{g}=\gamma^{l}$, and consumers are neural and become equally sensitive to gains and losses;

- if $\beta \in[0,1)$, then $\gamma^{g}>\gamma^{l}$, and consumers are gainseeking; if $\beta=0$, then $\gamma^{l}=0$, and consumers are insensitive to losses; and

- if $\beta>1$, then $\gamma^{g}<\gamma^{l}$, and consumers are loss-averse.

Gain-seeking consumers are observed in the market with promotion driven consumers or highly stockable products [3]. In line with this observation, we focus here on gain-seeking consumers and assume $\beta \in[0,1)$. In this setting, the demand becomes non-smooth due to being non-differentiable at the point $p_{n}=r_{n}$ (cf. Eq. (3a)-(3b)). Other scenarios (neutral and loss averse consumers) require different modelling and optimisation procedures and are therefore not considered here.

\section{Short-term revenue}

Suppose a decision maker aims to optimise the short-term revenue, then the optimal price choices are provided through

$$
p_{n}^{*}=\arg \max _{p_{n} \in[0, U]} \Pi=p_{n} d\left(p_{n}, r_{n}\right) .
$$

Lemma 1: The reference price evolves via

$r_{n+1}= \begin{cases}\alpha r_{n}+(1-\alpha) \frac{\gamma^{l} r_{n}+b}{2\left(a+\gamma^{l}\right)}, & \text { if } r_{n} \leq R \leq R_{U} \\ \alpha r_{n}+(1-\alpha) \frac{\gamma^{g} r_{n}+b}{2\left(a+\gamma^{g}\right)}, & \text { if } R<r_{n}, R \leq R_{U}, \text { and } \\ \alpha r_{n}+(1-\alpha) U & \text { if } R>R_{U}\end{cases}$

where $R=\frac{b}{a+\sqrt{\left(a+\gamma^{g}\right)\left(a+\gamma^{l}\right)}}, R_{U}=\frac{2\left(a+\gamma^{l}\right) U-b}{\gamma^{l}}$, cf. [3].

\section{Long-term revenue}

When a decision maker focuses on the long-term revenue, the optimal price choices are determined by the following equation

$$
\max _{p_{n} \in[0, U]} \sum_{n=0}^{\infty} \omega^{n} p_{n} d\left(p_{n}, r_{n}\right)
$$

where $\omega \in(0,1]$ represents a discount rate, which is a rate to evaluate the present cash value of the discounted future revenue [3].

Numerical approximation $p_{n}^{*}$ of Eq. (7) here is based on dynamic programming, which is described in more detail in [3], [9]. Plugging $p_{n}^{*}$ into Eq. (1), we get a map that describes the evolution of the reference price for the long-term revenue scenario.

Bifurcation diagrams summarise the entire behaviour of the system as it changes a significant parameter [17]. Bifurcation diagrams can analyse the change in the amplitude of the steady-state solutions as well as the change in the complexity of the solution. However, bifurcation diagrams may fail to 


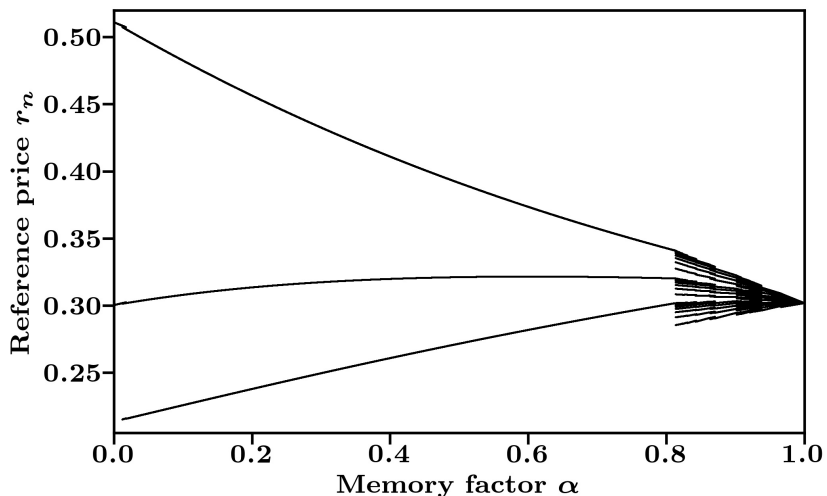

Fig. 1. Bifurcation diagram of reference price against memory factor in the case of optimising the short-term revenue.

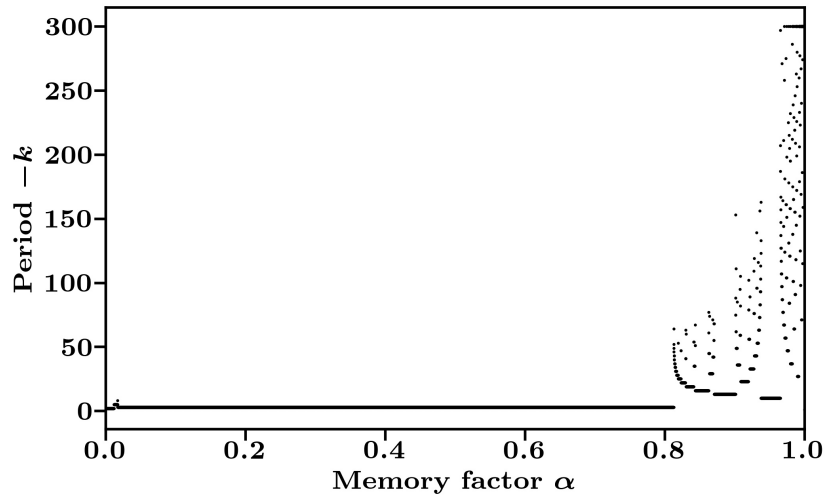

Fig. 2. Period diagram of reference price against memory factor in the case of optimising the short-term revenue.

detect the change in dynamics for border collision bifurcations [18]. Period diagrams record the period of solutions with the changing bifurcation parameter [19]. Period diagrams allows examining the robustness of steady-state solutions and allows comparing the optimisations with the short-term and the longterm revenue.

To generate the bifurcation and period diagrams, we assume the following parameters to be constant [3]:

$$
b=582, a=569.4, \gamma^{g}=2671.2, \omega=0.9, U=1,
$$

and choose the memory factor $\alpha$ and $\beta$ as the bifurcation parameter, respectively.

\section{DYNAMICS IN THE SHORT-TERM REVENUE STRATEGY}

Next the dynamic behaviour of the reference price as evolved via Eq. (6) is investigated through numerical simulations. We focus on the reference price rather than the optimal price choices for two reasons. From Eq. (6), the dynamic behaviour of the optimal price is in line with that of the reference price. Also, the evolution of the reference price provides critical information for a regulator to identify the behaviour of a company in the market.

\section{A. Variation of the memory rate $\alpha$}

In this section the relative sensitivity $\beta=0$ is fixed and the memory rate $\alpha$ is varied which would be a conventional way of studying this type of equation [3].

Fig. 1 depicts the results in form of a bifurcation diagram. When $\alpha \leq 0.813$, the reference price converges to three branches, however, when $\alpha \in(0.813,1]$, more branches with the decreasing amplitude of reference price emerge until a constant value is reached. Jumps in the bifurcation diagram can indicate the occurrence of either subcritical Hopf bifurcation or boder collision bifurcation [12], [20].

Fig. 2 depicts a period diagram. Except for a small interval $\alpha \in[0.012,0.017]$ where the period changes from 3 to 8 and then 5 , the number of periods remains constant at 3 . Complex changes in periodic behaviour emerge from around $\alpha=0.813$ up to unity, indicating the occurrence of border collision bifurcations [16], [21].

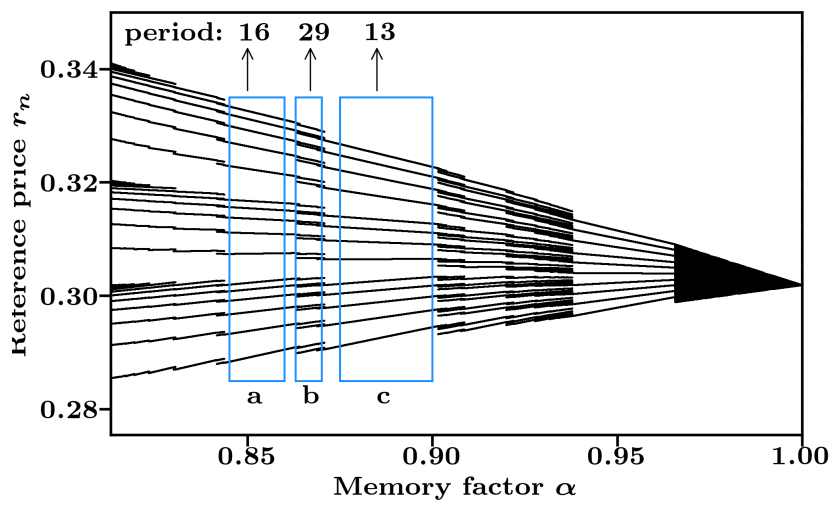

Fig. 3. Bifurcation diagram of reference price against the memory factor in the case of optimising the short-term revenue. Zoom in of Fig. 1. The branches in the window marked as ' $b$ ' are an overlap of its 'left' side branches marked as 'a' and its 'right' side branches marked as 'c'.

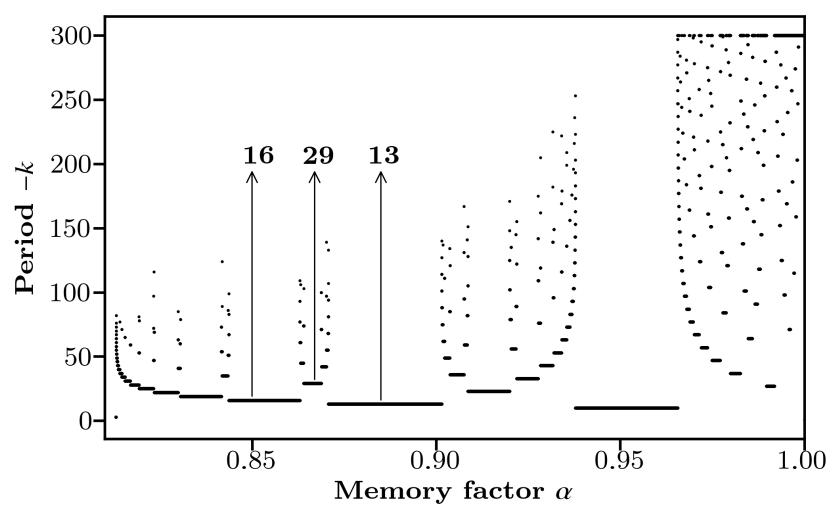

Fig. 4. Period diagram of reference price against the memory factor in the case of optimising the short-term revenue. Zoom-in of Fig. 2 shows period adding cascades. Between the period-16 and the period-13 solutions, its sum, a period-29 solution, emerges.

To investigate the dynamics in more detail as $\alpha \in(0.813,1]$, we zoom into both, the bifurcation diagram of Fig. 1 and the 
period diagram of Fig. 2. The results are shown in Fig. 3 and Fig. 4.

Fig. 3 shows overlaps of branches. The window marks with ' $b$ ' is the result of an overlap of branches in ' $a$ ' with those in c. Fig. 4 reveals in detail these period adding cascades [22]. The period adding cascades within the period-13, period-16 and period-29 window are called period adding bifurcations as a result of border collisions [22]-[24]. According to this adding rule, there is a period- 45 window between the period- 16 and the period-29, and so forth.

For the short-term revenue, the reference price is robust to a change in the memory rate of consumers such that $\alpha<0.813$, leads to a robust period- 3 optimal pricing strategy. However, as $\alpha>0.813$, period adding bifurcations emerge. This leads to the maximised revenue generated from a periodic pricing strategy with a frequent change in its period.

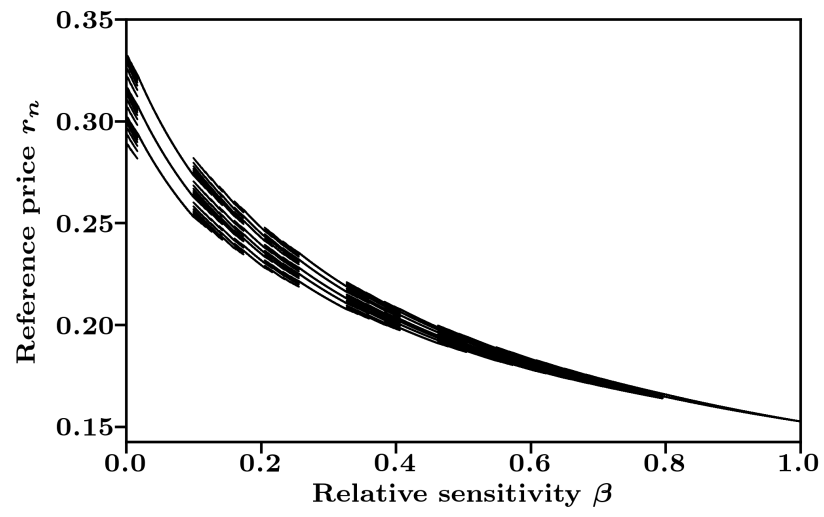

Fig. 5. Bifurcation diagram of reference price against relative sensitivity of consumers in the case of optimising the long-term revenue.

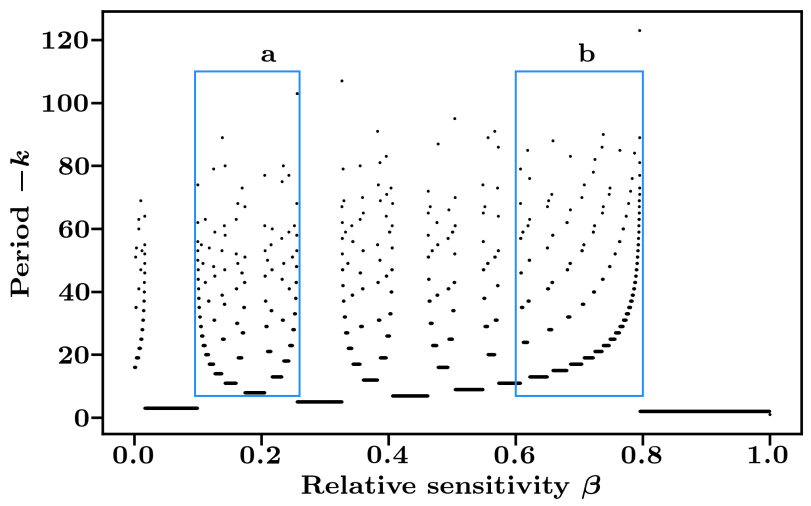

Fig. 6. Period diagram of the reference price against the relative sensitivity in the case of optimising the long-term revenue. The the window marked with an 'a' highlights a U-cascade which contains pairs of opposed period adding bifurcation cascades; the window marked by ' $b$ ' represents an S-cascade which only consists of period adding cascades of increasing order.

\section{B. Variation of the relative sensitivity $\beta$}

Next we set the the memory factor to be $\alpha=0.85$, and alter the relative sensitivity $\beta$ as shown in Fig. 5. As $\beta$ increases, the amplitude of the reference price decreases until a constant value is reached. Again, cascades of period adding bifurcations are observed. In the following Fig. 6 illustrates two scenarios of period adding cascades as $\beta$ increases:

1) For the window marked as ' $a$ ', the lowest period lies in the middle of the period adding cascade which is called in the following a U-cascade. In a U-cascade the period of its right-hand side is greater than that of left-hand side.

2) For the window marked with 'b', we observe an Scascade of which period adding cascades have layers of increasing periods.

For the short-term revenue strategy, period adding bifurcations are observed only when $\beta<0.795$. As the relative sensitivity to losses and gains approaches unity, the reference price becomes a period- 2 solution, which is robust to the change in the relative sensitivity.

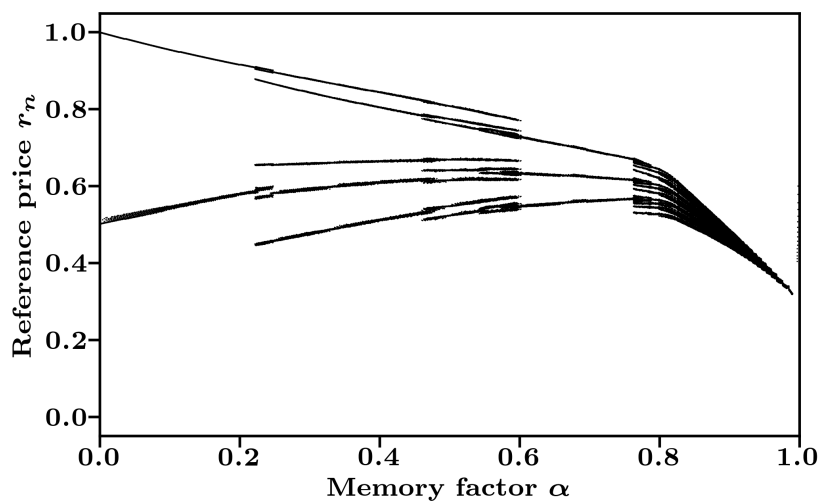

Fig. 7. Bifurcation diagram of reference price against the memory factor in the case of optimising the long-term revenue.

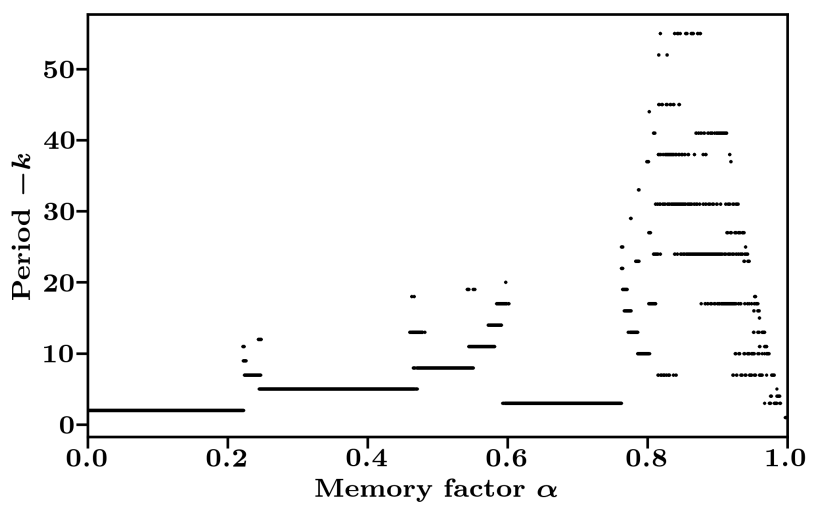

Fig. 8. Period diagram of reference price against the memory factor in the case of optimising the long-term revenue.

\section{DYNAMICS IN THE LONG-TERM REVENUE STRATEGY}

This section investigate the nonlinear dynamics of reference price for a decision maker aiming to optimise the long-term revenue. 


\section{A. Variation of memory rate $\alpha$}

The parameters used here are the same as those in section III-A but a different objective function is used: The decision maker obtains the optimal price from the solutions to Eq. (7).

Fig. 7 depicts the bifurcation diagram; again bifurcations in branches add until $\alpha$ reaches a value of about 0.6 , where the branches start merging into three main branches. This convergence is followed by the emergence of many branches and the shrinking amplitude until a constant value is reached.

However, when comparing the $y$-axis of Fig. 1 and Fig. 7, we find that for the same $\alpha$, the reference price in the case of the long-term revenue can be higher than in the case of the short-term revenue. The result is consistent with observations in [5]. A company chooses usually a higher sales price to increase future reference prices and thereby to grow its longterm revenue.

Fig. 8 depicts the change in dynamics with $\alpha$. As $\alpha \in$ $(0,0.222]$, the reference price becomes a period- 2 solution. As $\alpha \in(0.222,0.592)$, period adding bifurcations occur.

However, comparisons between the short-term (cf. Fig. 2) and the long-term revenue (cf. Fig. 8) in the interval $\alpha \in$ $[0,0.813)$ shows the robustness of the short-term revenue. The former is almost a period-3 solution and no evident period adding bifurcations. In contrast, the latter observes evident period adding bifurcations.

From the comparisons between the behaviour of the reference price regarding the amplitude and the period, a decision maker can charge a higher price when aiming to the long-term revenue. However, opposed to the short-term revenue strategy for values $\alpha<0.813$, the consideration of the long-term revenue renders scenarios that the reference price becomes prone to period adding bifurcations.

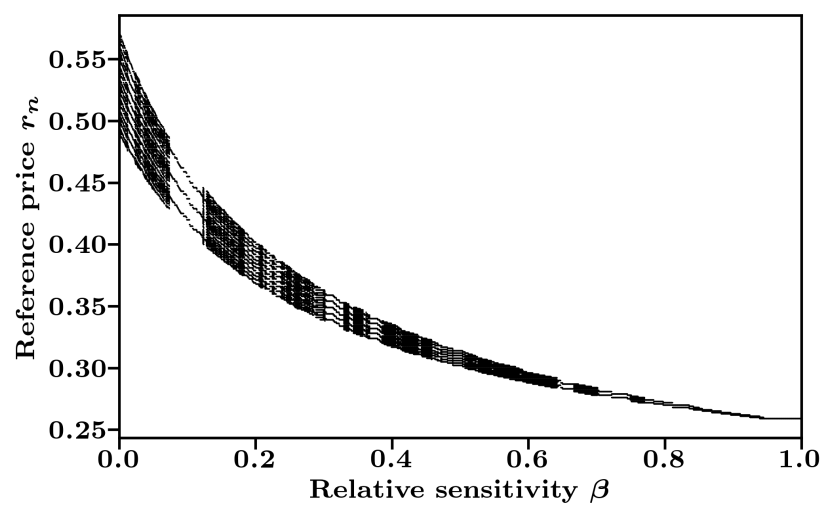

Fig. 9. Bifurcation diagram of reference price against the relative sensitivity in the case of optimising the long-term revenue.

\section{B. Variation of relative sensitivity $\beta$}

The parameters are the same as section III-B. The optimal price is approximated according to Eq. (7).

Fig. 9 shows the bifurcation diagram as the relative sensitivity changes. Similar to Fig. 5, the branches show bifurcation adding cascades. Comparisons between Fig. 9 and Fig. 5

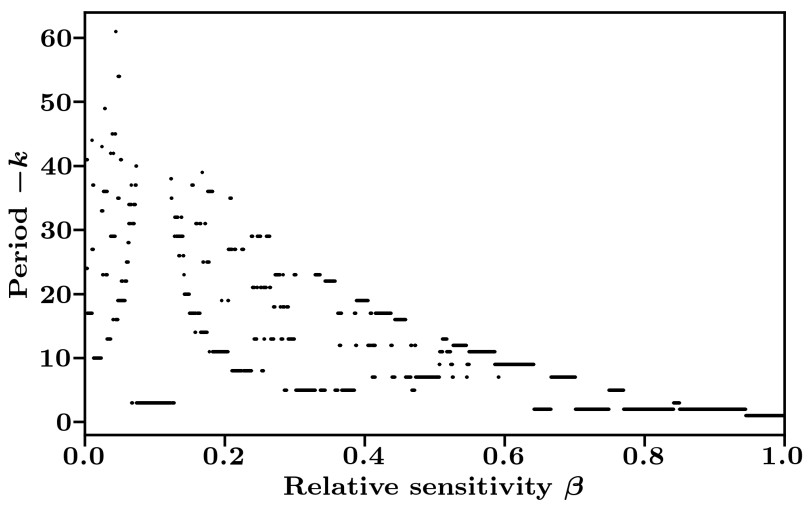

Fig. 10. Period diagram of reference price against the relative sensitivity in the case of optimising the long-term revenue.

further validate our observations: we can achieve a higher reference price for the long-term revenue strategy than in the short-term revenue strategy. Also, U-cascades shown in Fig. 10 is not so obvious as in Fig. 6. This observation indicates that the consideration of the long-term revenue reduce the variation in the period of the periodic reference price.

A reduced variation is due to a clairvoyant perspective in solving the optimisation problem in the case of the longterm revenue. The underlying assumption is that the system does not follow any drift terms, evolves according to given equations and that the 'maximal generating revenue' price path is chosen. The decision maker has full information of the market evolution.

This information helps the decision maker to reduce unnecessary transitions to steady-state solutions. As a result, the long-term consideration reduces the amplitude of the change in dynamics when the period adding bifurcations emerge both in the short-term and the long-term revenue strategy.

\section{CONClusion}

We have studied bifurcation and period diagrams to examine the nonlinear dynamics in a dynamic pricing model considering reference effects. Comparisons between a short-term and a long-term revenue strategy have been conducted. Period adding bifurcations are observed as the market environment changes. According to the order of the left and the right hand side of the period adding cascades, we classify two types of cascades: the U-cascade and the S-cascade according to Granados and Huguet [25]. These two types of cascades enable us to identify different bifurcation structures which belong to different revenue strategies. The classification of cascades can further be used to characterise period adding bifurcations into different evolution of rotation numbers.

The consideration of optimising the long-term revenue has two advantages over optimising the short-term revenue. A decision maker could charge temporarily a higher price to increase the reference price. Also, the long-term revenue strategy induces a less variation in the period. Further, this reduced variability lowers the amplitude of the period of the reference price. 
However, optimising the long-term revenue breaks a supposedly robust pricing strategy in some situations (here $\alpha \in$ $(0.222,0.592))$. In this situation, the short-term revenue generates a robust periodic solution with a fixed period, which avoids period adding bifurcations. Knowing of such a robust period would enable a decision maker to reduce price volatility and manage consumer price expectations.

Our focus is on the periodic solution of the reference price and solutions with a period smaller than 300 using period diagrams. This limitation, however, renders it difficult to distinguish between higher periodic, quasi-periodic or chaotic solutions. Symbolic analyses or order pattern recurrent plots and associated quantifiers may provide further condensation of the complex information contained in bifurcation diagrams [26].

\section{REFERENCES}

[1] A. Mehra, S. Kumar, and J. S. Raju, "Competitive strategies for brickand-mortar stores to counter showrooming," Management Science, 2017.

[2] J. Li, N. Granados, and S. Netessine, "Are consumers strategic? structural estimation from the air-travel industry," Management Science, vol. 60, no. 9, pp. 2114-2137, 2014.

[3] Z. Hu, X. Chen, and P. Hu, "Dynamic pricing with gain-seeking reference price effects," Operations Research, vol. 64, no. 1, pp. 150$157,2016$.

[4] T. Mazumdar, S. P. Raj, and I. Sinha, "Reference price research: Review and propositions," Journal of marketing, vol. 69 , no. 4, pp. 84-102, 2005.

[5] I. Popescu and Y. Wu, "Dynamic pricing strategies with reference effects," Operations Research, vol. 55, no. 3, pp. 413-429, 2007.

[6] P. K. Kopalle, A. G. Rao, and J. L. Assuncao, "Asymmetric reference price effects and dynamic pricing policies," Marketing Science, vol. 15, no. 1 , pp. 60-85, 1996.

[7] P. K. Kopalle, P. Kannan, L. B. Boldt, and N. Arora, "The impact of household level heterogeneity in reference price effects on optimal retailer pricing policies," Journal of Retailing, vol. 88, no. 1, pp. 102114, 2012.

[8] N. Mizik and R. Jacobson, "Myopic marketing management: Evidence of the phenomenon and its long-term performance consequences in the seo context," Marketing Science, vol. 26, no. 3, pp. 361-379, 2007.

[9] X. Chen, P. Hu, and Z. Hu, "Efficient algorithms for the dynamic pricing problem with reference price effect," Management Science, vol. 63, no. 12 , pp. 4389-4408, 2017.

[10] R. L. Phillips, Pricing and revenue optimization. Stanford University Press, 2005

[11] K. T. Talluri and G. J. Van Ryzin, The theory and practice of revenue management. Springer Science \& Business Media, 2006, vol. 68.

[12] S. Yin, J. Ji, G. Wen, and X. Wu, "Use of degeneration to stabilize near grazing periodic motion in impact oscillators," Commun. Nonlinear Sci. Numer. Simul., vol. 66, pp. 20-30, 2019.

[13] R. Vasconcellos and A. Abdelkefi, "Nonlinear dynamical analysis of an aeroelastic system with multi-segmented moment in the pitch degreeof-freedom," Communications in Nonlinear Science and Numerical Simulation, vol. 20, no. 1, pp. 324-334, 2015.

[14] A. Panchuk, I. Sushko, and F. Westerhoff, "A financial market model with two discontinuities: Bifurcation structures in the chaotic domain," Chaos: An Interdisciplinary Journal of Nonlinear Science, vol. 28, no. 5, p. 055908, 2018.

[15] A. K. Naimzada and M. Pireddu, "Fashion cycle dynamics in a model with endogenous discrete evolution of heterogeneous preferences," Chaos: An Interdisciplinary Journal of Nonlinear Science, vol. 28, no. 5 , p. 055907, 2018.

[16] L. Gardini, I. Sushko, and K. Matsuyama, "2d discontinuous piecewise linear map: Emergence of fashion cycles," Chaos: An Interdisciplinary Journal of Nonlinear Science, vol. 28, no. 5, p. 055917, 2018.

[17] J. C. Sprott and J. C. Sprott, Chaos and time-series analysis. Citeseer, 2003, vol. 69 .
[18] F. Nazarimehr, S. Jafari, S. M. R. Hashemi Golpayegani, M. Perc, and J. C. Sprott, "Predicting tipping points of dynamical systems during a period-doubling route to chaos," Chaos: An Interdisciplinary Journal of Nonlinear Science, vol. 28, no. 7, p. 073102, 2018.

[19] B. Futter, V. Avrutin, and M. Schanz, "The discontinuous flat top tent map and the nested period incrementing bifurcation structure," Chaos, Solitons \& Fractals, vol. 45, no. 4, pp. 465 - 482, 2012.

[20] Z. Zhang, S. Oberst, and J. Lai, "A non-linear friction work formulation for the analysis of self-excited vibrations," Journal of Sound and Vibration, 2018.

[21] P. Jain and S. Banerjee, "Border-collision bifurcations in onedimensional discontinuous maps," International Journal of Bifurcation and Chaos, vol. 13, no. 11, pp. 3341-3351, 2003.

[22] A. Granados, L. Alsedà, and M. Krupa, "The period adding and incrementing bifurcations: from rotation theory to applications," SIAM Rev., vol. 59, no. 2, pp. 225-292, 2017.

[23] D. Dutta and J. Bhattacharjee, "Period adding bifurcation in a logistic map with memory," Physica D: Nonlinear Phenomena, vol. 237, no. 23, pp. 3153-3158, 2008.

[24] L. Gardini, V. Avrutin, and I. Sushko, "Codimension-2 border collision bifurcations in one-dimensional discontinuous piecewise smooth maps," Int. J. Bifurcation Chaos, vol. 24, no. 02, p. 1450024, 2014.

[25] A. Granados and G. Huguet, "Gluing and grazing bifurcations in periodically forced 2-dimensional integrate-and-fire models," Communications in Nonlinear Science and Numerical Simulation, 2018.

[26] S. Lu, S. Oberst, G. Zhang, and Z. Luo, "Novel order patterns recurrence plot-based quantification measures to unveil deterministic dynamics from stochastic processes," in ITISE 2018 (International conference on Time Series and Forecasting), September 19th-21th, 2018. 